\title{
Pneumonia Management in Nursing Homes: Findings from a CMS Demonstration Project
}

J Gen Intern Med 36(2):570-2

DOI: $10.1007 / \mathrm{s} 11606-020-05885-0$

(c) Society of General Internal Medicine 2020

\section{INTRODUCTION}

For nursing facility residents, potentially avoidable hospitalizations (PAHs) can lead to adverse outcomes such as delirium, nosocomial infections, and decline in function. PAHs are also costly to the Medicare program. ${ }^{1,2}$ The Indiana-based Optimizing Patient Transfers, Impacting Medical Quality and Improving Symptoms: Transforming Institutional Care (OPTIMISTIC) project is one of six demonstration projects across the country awarded a contract from the Centers for Medicare and Medicaid (CMS) to reduce PAHs for long-stay nursing facility residents. ${ }^{1}$ All OPTIMISTIC nursing home residents have Medicare; approximately 2/3 also have Medicaid coverage.

OPTIMISTIC is targeting six specific diagnoses associated with PAHs: dehydration, cellulitis, exacerbations of heart failure or obstructive pulmonary disease, urinary tract infection, and pneumonia. ${ }^{1}$ Project facilities are eligible for enhanced reimbursement through Medicare Part B codes when one of the six conditions is treated in the facility, instead of transferring the resident to the hospital. In addition to the enhanced reimbursement, 17 of the facilities also have dedicated project clinical staff on-site to support facility staff (clinical + payment) and 23 only receive the enhanced reimbursement (payment only). In analyses of the OPTIMISTIC nursing facilities with on-site support, PAHs were reduced by $1 / 3 .^{3}$

More than $30 \%$ of PAHs of nursing facility residents are for pneumonia, making pneumonia one of the top five PAHassociated diagnoses. ${ }^{2}$ Using OPTIMISTIC data, we are uniquely positioned to describe current treatment for episodes of pneumonia in nursing facilities. The goal of this case series is to describe the management of episodes of pneumonia in nursing facilities participating in the OPTIMISTIC project.

\section{CASE SERIES}

\section{Methods}

To be eligible for this study, selected cases had to meet the OPTIMISTIC project inclusion criteria (length of stay $>$

Received January 3, 2020

Accepted April 28, 2020

Published online June 3, 2020
100 days; Medicare fee-for-service coverage). Of the 40 participating nursing facilities, the monthly pneumonia incidence ranged from 0 to 9 cases. Those with high incidence of pneumonia episodes treated in house (5 or more episodes) were reviewed, and 3-4 cases from each facility were randomly selected for review (https://www.random.org/). Data sources included electronic and paper medical records, lab results, chest x-ray readings, and OPTIMISTIC project data managed in REDCap.

\section{Results}

Cases for review were drawn from 7 facilities from November 2017 to April 2018, for a total of 41 episodes of pneumonia. Most residents (Table 1) were over the age of $65(90.2 \%)$, female (63.4\%), and Caucasian (92.7\%). None of the cases of nursing facility acquired pneumonia had sputum or blood cultures submitted to laboratories (Table 2 part a). $58.3 \%$ of the cases had a CURB-65 score that was greater than or equal to 2 , which is a score used to estimate need for inpatientlevel care. ${ }^{4}$ Among cases reviewed, $70.7 \%$ of the residents had 3 or more comorbidities that increased their risk for developing pneumonia while $95.1 \%$ of them were on a medication that increases the risk for pneumonia (Table 1).

All pneumonia cases were treated with antibiotics and more than half $(53.7 \%)$ were treated with a fluoroquinolone (Table 2). The duration of antibiotic administration was greater than 7 days in $39.0 \%$ of cases reviewed. Supportive medical care was implemented in all cases in both the clinical and the clinical plus payment buildings including more frequent nursing assessments, vital sign monitoring, and support for oral hydration. Most (87.8\%) of the residents were able to remain in place in the facility for treatment although 3 residents $(7.3 \%)$ were hospitalized, one $(2.4 \%)$ was transferred to hospice, and one person who did not have aggressive goals of care died.

\section{DISCUSSION}

This study demonstrates that despite their multi-morbidity and frailty, nursing facility residents can be safely treated for pneumonia in a nursing facility when supportive medical care is provided. This includes more frequent monitoring and assessments during the episode of acute illness. Limitations of 
Table 1 Population and Facility Demographics

\begin{tabular}{|c|c|c|c|}
\hline \\
\hline \multicolumn{4}{|l|}{$N=41$} \\
\hline & Total & Clinical + payment & Payment only \\
\hline Age $\geq 65$ & $90.2(37)$ & & \\
\hline Gender (female) & $63.4(26)$ & & \\
\hline Race (Caucasian) & $92.7(38)$ & & \\
\hline 3 or more risk-related comorbidities & $70.7(29)$ & & \\
\hline Pneumonia recurrence (previous 6 months) & $51.2(21)$ & & \\
\hline Taking at least 1 medication that may increase risk of pneumonia ${ }^{\dagger}$ & $95.1(39)$ & & \\
\hline Nursing facilities & & & \\
\hline$n=7$ ( 3 facilities $=$ payment only; $4=$ payment plus clinical support $)$ & \# (range) & & \\
\hline & Total & Clinical + payment & Payment only \\
\hline Mean number of certified beds & $149.86(111-192)$ & 156.75 & 140.67 \\
\hline Proportion of OPTIMISTIC eligible residents & $0.44(0.29-0.56)$ & $0.45(0.29-0.56)$ & $0.43(0.32-0.53)$ \\
\hline Average Medicare star rating during the study period & 4.0 & 3.8 & \\
\hline Mean number of pneumonia cases during study period & 12.43 & 10.00 & 15.67 \\
\hline
\end{tabular}

${ }^{\dagger}$ Proton pump inhibitors, $\mathrm{H}_{2}$ receptor antagonists, inhaled glucocorticoids, sedatives, typical/atypical antipsychotics, warfarin, immunosuppressive medication

this case series include review of a limited number of cases and lack of context such as the antibiogram of the facilities.

Despite FDA warnings and treatment recommendations, fluoroquinolone use was high and treatment duration was longer than recent guidelines recommend. Increasing use of rapid testing may enable nursing facility providers to better tailor treatments to pathogens. ${ }^{5}$ Nursing facility quality improvement initiatives should target antibiotic stewardship and employ clinical practice guidelines to treat this highly vulnerable population in place.

Table 2 Diagnosis, Treatment, and Outcomes: Mean (Percent)

\begin{tabular}{|c|c|c|c|}
\hline \multicolumn{4}{|l|}{ a: Signs and symptoms present at diagnosis } \\
\hline CMS diagnostic criteria for pneumonia & Total \% $(N)$ & Clinical + payment & Payment only \\
\hline Fever $>100^{\circ} \mathrm{F}$ or $\geq 2^{0}$ above baseline & $17.1(7)$ & $14.6(6)$ & $2.4(1)$ \\
\hline Oxygen saturation $<92 \%$ on room air or on resident's usual oxygen settings & $63.4(26)$ & $41.5(17)$ & $22.0(9)$ \\
\hline Respiratory rate $>24$ breaths/min & $4.9(2)$ & $2.4(1)$ & $2.4(1)$ \\
\hline Evidence of at least one focal pulmonary consolidation on exam* & $97.6(40)$ & $53.7(22)$ & $43.9(18)$ \\
\hline Chest X-ray confirming new pulmonary infiltrate & $80.5(33)$ & $39.0(16)$ & $41.5(17)$ \\
\hline \multicolumn{4}{|l|}{ Symptoms } \\
\hline Cough & $85.4(35)$ & $51.2(21)$ & $34.1(14)$ \\
\hline Sputum production & $51.2(21)$ & $29.3(12)$ & $22.0(9)$ \\
\hline Shortness of breath & $39.0(16)$ & $19.5(8)$ & $19.5(8)$ \\
\hline \multicolumn{4}{|l|}{ Other diagnostic criteria } \\
\hline Sputum culture, blood culture or PCR performed & $0.0(0)$ & $0.0(0)$ & $0.0(0)$ \\
\hline CURB-65 scores $0-1 * *$ & $41.5(17)$ & $24.4(10)$ & $17.1(7)$ \\
\hline CURB-65 score $=2$ & $34.1(14)$ & $17.1(7)$ & $17.1(7)$ \\
\hline CURB- 65 score $\geq \overline{3}$ & $24.2(10)$ & $14.6(6)$ & $9.8(4)$ \\
\hline Diagnosis by physician & $39.0(16)$ & $19.5(8)$ & $19.5(8)$ \\
\hline Diagnosis by advanced practice provider & $61.0(25)$ & $36.6(15)$ & $24.4(10)$ \\
\hline \multicolumn{4}{|l|}{ b: Treatment and outcomes } \\
\hline Antibiotic treatment & Total \% $(N)$ & Clinical + payment & Payment only \\
\hline Fluoroquinolone & $53.7(22)$ & $29.3(12)$ & $24.4(10)$ \\
\hline Penicillin combination & $26.8(11)$ & $19.5(8)$ & $7.3(3)$ \\
\hline Cephalosporin (any generation) & $24.4(10)$ & $12.2(5)$ & $12.2(5)$ \\
\hline Tetracycline & $12.2(5)$ & $7.3(3)$ & $4.9(2)$ \\
\hline Macrolide & $12.2(5)$ & $7.3(3)$ & $4.9(2)$ \\
\hline Duration of antibiotic treatment ( 7 days or less) & $61.0(25)$ & $36.6(15)$ & $24.4(10)$ \\
\hline \multicolumn{4}{|l|}{ Other treatments } \\
\hline Bronchodilator & $82.9(34)$ & $48.8(20)$ & $34.1(14)$ \\
\hline Corticosteroids & $22.0(9)$ & $14.6(6)$ & $7.3(3)$ \\
\hline Oxygen supplementation & $70.7(29)$ & $46.3(19)$ & $24.4(10)$ \\
\hline Supportive cares for pneumonia*** & $100.0(41)$ & $100.0(23)$ & $100.0(18)$ \\
\hline Supportive care treatment longer than billing episode length & $78.0(32)$ & $39.0(16)$ & $39.0(16)$ \\
\hline \multicolumn{4}{|l|}{ Outcomes } \\
\hline Stabilized in the nursing facility & $87.8(36)$ & $51.2(21)$ & $36.6(15)$ \\
\hline Hospitalized & $7.3(3)$ & $0.0(0)$ & $7.3(3)$ \\
\hline Transferred to hospice & $2.4(1)$ & $2.4(1)$ & $0.0(0)$ \\
\hline Died & $2.4(1)$ & $2.4(1)$ & $0.0(0)$ \\
\hline
\end{tabular}

*Rales, rhonchi, decreased breath sounds, and dullness to percussion

$* * C U R B-65$ score between 0 and 1 outpatient care is recommended, score $=2$ indicates hospital stay may be indicated, score $\geq 3$ indicates severe pneumonia that should be managed in the hospital

***Hydration, frequent vitals, and close monitoring 
Jennifer L. Carnahan, $M D, M P H, M A^{1,2,3}$

Andrew J. Shearn, $B A^{3}$

Kristi M. Lieb, $M D^{3}$

Kathleen T. Unroe, $M D, M H A^{1,2,3}$

${ }^{1}$ Indiana University Center for Aging Research,

Indianapolis, IN, USA

${ }^{2}$ Regenstrief Institute Inc,

Indianapolis, IN, USA

${ }^{3}$ Indiana University School of Medicine,

Indianapolis, IN, USA

Corresponding Author: Jennifer L. Carnahan, MD, MPH, MA; Indiana University School of Medicine, Indianapolis, IN, USA (e-mail: jenncarn@iupui.edu).

Funding Information Dr. Carnahan reports that this publication was made possible with support from Grant Numbers KL2TRO02530 (A Carroll, PI) and UL1TRO02529 (A. Shekhar, PI) from the National Institutes of Health, National Center for Advancing Translational Sciences, Clinical and Translational Sciences Award. OPTIMISTIC is supported by the Centers for Medicare and Medicaid Services (CMS) of the U.S. Department of Health and Human Services (HHS) as part of an award totaling $\$ 16,545,692$ with $0 \%$ percentage financed with nongovernmental sources.

\section{Compliance with Ethical Standards:}

Conflict of Interest: Dr. Kathleen Unroe is the CEO and Founder of Probari, a business intended to disseminate the OPTIMISTIC clinical care model.

Disclaimer: The contents are those of the authors and do not necessarily represent the official views of, nor an endorsement, by CMS, HHS, or the US government.

\section{REFERENCES}

1. Unroe KT, Fowler NR, Carnahan JL, et al. Improving Nursing Facility Care Through an Innovative Payment Demonstration Project: Optimizing Patient Transfers, Impacting Medical Quality, and Improving Symptoms: Transforming Institutional Care Phase 2. J Am Geriatr Soc 2018;66(8):1625-1631.

2. Walsh EG, Wiener JM, Haber S, Bragg A, Freiman M, Ouslander JG. Potentially avoidable hospitalizations of dually eligible Medicare and Medicaid beneficiaries from nursing facility and Home- and CommunityBased Services waiver programs. J Am Geriatr Soc 2012;60(5):821-829.

3. Ingber $\mathbf{M J}$, Feng $\mathbf{Z}$, Khatutsky $\mathbf{G}$, et al. Evaluation of the Initiative to Reduce Avoidable Hospitalizations among Nursing Facility Residents: Final Report. Research Triangle Institute;2017.

4. Lim WS, van der Eerden $\mathbf{M M}$, Laing $\mathbf{R}$, et al. Defining community acquired pneumonia severity on presentation to hospital: an international derivation and validation study. Thorax. 2003;58(5):377-382.

5. Jump RLP, Crnich CJ, Mody L, Bradley SF, Nicolle LE, Yoshikawa TT. Infectious Diseases in Older Adults of Long-Term Care Facilities: Update on Approach to Diagnosis and Management. J Am Geriatr Soc 2018;66(4):789-803.

Publisher's Note: Springer Nature remains neutral with regard to jurisdictional claims in published maps and institutional affiliations. 